\title{
Molecular cloning, expression and IgE-immunoreactivity of phospholipase A1, a major allergen from Polybia paulista (Hymenoptera: Vespidae) venom
}

\author{
Amilcar Perez-Riverol ${ }^{a}$, Franco Dani Campos Pereira ${ }^{\mathrm{a}, \mathrm{b}}$, Alexis Musacchio Lasa ${ }^{\mathrm{c}}$, \\ Luis Gustavo Romani Fernandes ${ }^{\mathrm{d}}$, José Roberto Aparecido dos Santos-Pinto ${ }^{\mathrm{e}}$, \\ Débora Lais Justo-Jacomini ${ }^{\mathrm{a}}$, Gabriel Oliveira de Azevedo ${ }^{\mathrm{f}}$, Murilo Luiz Bazon ${ }^{\mathrm{a}}$, \\ Mario Sergio Palma e , Ricardo de Lima Zollner ${ }^{\mathrm{d}}$, Márcia Regina Brochetto-Braga a, g, * \\ ${ }^{a}$ Laboratório de Biologia Molecular de Artrópodes-LBMA-IBRC-UNESP (Univ Estadual Paulista), Av. 24-A, $n^{\circ}$ 1515, CEP 13506-900, Bela Vista, Rio Claro, SP, \\ Brazil \\ ${ }^{\mathrm{b}}$ Laboratório de Mutagênese Ambiental, Avenida 24-A, $n^{\circ}$ 1515, Bela Vista, Rio Claro, São Paulo, CEP 13506-900, Brazil \\ ${ }^{\mathrm{c}}$ Center for Genetic Engineering and Biotechnology, Biomedical Research Division, System Biology Department, Ave. 31, e/ 158 and 190, P.O. Box 6162, \\ Cubanacan, Playa, Havana, 10600, Cuba \\ d Laboratório de Imunologia Translacional, Faculdade de Ciências Médicas, FCM, Universidade Estadual de Campinas-UNICAMP, Rua Vital Brasil, ${ }^{\circ}$ 300, CEP \\ 13083-887, Cidade Universitária “Zeferino Vaz", Campinas, SP, Brazil \\ e Centro de Estudos de Insetos Sociais-CEIS-IBRC-UNESP (Univ Estadual Paulista), Av. 24-A, $n^{\circ} 1515$, CEP 13506-900, Bela Vista, Rio Claro, SP, Brazil \\ ${ }^{\mathrm{f}}$ Instituto de Pesquisa em Bioenergia (IPBEN) (Univ Estadual Paulista), Av. 24-A, $n^{\circ} 1515$, CEP 13506-900, Bela Vista, Rio Claro, SP, Brazil \\ ${ }^{g}$ Centro de Estudos de Venenos e Animais Peçonhentos-CEVAP (Univ Estadual Paulista), Rua José Barbosa de Barros, 1780, Fazenda Experimental Lageado, \\ Botucatu 18610-307, SP, Brazil
}

\section{A R T I C L E I N F O}

\section{Article history:}

Received 20 June 2016

Received in revised form

5 September 2016

Accepted 3 November 2016

Available online 5 November 2016

\section{Keywords:}

Polybia paulista

Venom

Allergy

Diagnosis

Recombinant phospholipase A1

Immunoglobulin E (IgE)

\begin{abstract}
A B S T R A C T
Polybia paulista (Hymenoptera: Vespidae) is a clinically relevant social wasp that frequently causes stinging accidents in southeast Brazil. To date, diagnosis and specific immunotherapy (SIT) of allergy are based on the use of crude venom extracts. Production of recombinant forms of major allergens from P. paulista venom will improve diagnosis and SIT of allergic patients by reducing the incidence of crossreactivity and non-specific sensitization. Here, we describe the molecular cloning, heterologous expression, purification and IgE-mediated immunodetection of phospholipase A1 (Poly $p$ 1), a major allergen from $P$. paulista venom. The cDNA of Poly $p 1$ was extracted from venom glands and then cloned, and further expression of the recombinant allergen (rPoly $p$ 1) was achieved in Escherichia coli BL21 (DE3) cells. Purification of rPoly $p 1$ was performed using immobilized $\mathrm{Ni}^{2+}$ metal affinity chromatography. Also, a single-step chromatographic method allowed the purification of native Poly $p 1$ (nPoly $p 1$ ) from the wasp's venom glands. We used western blotting to evaluate IgE-reactivity of the sera from 10 $P$. paulista venom-allergic patients to rPoly $p 1$ and $\mathrm{nPoly} p$ 1. High levels of insoluble $\mathrm{rPoly} p 1$ were obtained during heterologous expression. After solubilization of inclusion bodies and purification of the recombinant protein, a unique band of $\sim 34 \mathrm{kDa}$ was detected in SDS-PAGE analysis. Allergen-specific IgE
\end{abstract}

Abbreviations: rPoly p 1, recombinant phospholipase A1 from P. paulista venom; nPoly $p$ 1, native phospholipase A1 from P. paulista venom; sIgE, specific IgE; PLA1, phospholipase A1; vPLA1s, vespid phospholipases A1; HVA, Hymenoptera venom allergy; SIT, specific immunotherapy; CRD, component-resolved diagnosis.

* Corresponding author. Laboratório de Biologia Molecular de Artrópodes-LBMAIBRC-UNESP (Univ Estadual Paulista), Av. 24-A, nº 1515, CEP 13506-900, Bela Vista, Rio Claro, SP, Brazil.

E-mail addresses: aperezriverol@gmail.com (A. Perez-Riverol), franko_mg@ hotmail.com (F.D. Campos Pereira), alexis.musacchio@cigb.edu.cu (A. Musacchio Lasa), luisgrf1982@gmail.com (L.G. Romani Fernandes), jrbio04@rc.unesp.br (J.R.A. Santos-Pinto), dmjacomini@gmail.com (D.L. Justo-Jacomini), gabriel. azevedo4@outlook.com (G. Oliveira de Azevedo), bazonmurilo@gmail.com (M.L. Bazon), mspalma@rc.unesp.br (M.S. Palma), zollner@unicamp.br (R.L. Zollner), mrbbraga@rc.unesp.br (M.R. Brochetto-Braga). 
(sIgE) from allergic patients' sera recognized rPoly $p 1, \mathrm{nPoly} p 1$ and crude venom extract to a similar extent. Our results showed that rPoly $p 1$ could be used for development of component-resolved diagnosis (CRD) and molecular-defined SIT of $P$. paulista venom allergy.

(C) 2016 Elsevier Ltd. All rights reserved.

\section{Introduction}

Hymenoptera (Apoidea, Vespidae, Formicidae) venom allergy is one of the most common causes of anaphylaxis worldwide, accounting for $1.5 \%-34.1 \%$ of the cases (Bilò, 2011). Because of the close coexistence of social Hymenoptera with human populations, an estimated $56 \%-94 \%$ of adults worldwide have been stung at least once in their lifetime (Jennings et al., 2010). After being stung, allergic patients experience local and/or systemic reactions, including life threatening anaphylaxis. The estimated prevalence of systemic reactions associated with Hymenoptera venom allergy (HVA) is $0.3 \%-8.9 \%$, of those cases, anaphylaxis occurs in $0.3 \%-$ 42.8\% (Bilò and Bonifazi, 2009).

In Brazil, despite the great number of clinically relevant social Hymenoptera and stinging accidents, specific diagnosis and immunotherapy rely on the use of whole venom extracts. These allergenic materials are associated with the occurrence of IgEmediated cross-reactivity during diagnosis, which could lead to non-specific sensitization and undesired side effects along the SIT (Ollert and Blank, 2015).

Several works have stated that CRD based on the use of panels of recombinant allergens significantly improves specific identification of culprit venom in patients diagnosed with double sensitization (Spillner et al., 2014; Perez-Riverol et al., 2015). To date, mainly recombinant forms of phospholipase A2 (Api $m$ 1) and hyaluronidase (Api m 2) from Apis mellifera, along with phospholipase A1 (Ves $v 1$ ) and antigen 5 (Ves $v 5$ ) from Vespula vulgaris, have been used to distinguish bee and/or wasp sensitization in allergic patients. Additionally, other bee venom allergens such as phosphatase (Api $\mathrm{m}$ 3 ) and icarapin (Api $m$ 10) have recently been shown to improve detection of honeybee venom (HBV) sensitization in allergic patients (Frick et al., 2015). Müller et al. (2012) reported that only 47\% of 76 patients previously diagnosed as double positive for whole venom extract of $A$. mellifera and of $V$. vulgaris reacted with $\mathrm{rVes} v 1$, rVes $v 5$, and rApi $m 1$ during specific IgE (sIgE) detection. The combined use of natural and recombinant forms of Ves $v 1$, Ves $v 5$, phospholipase A1 (Pol d 1) and antigen 5 (Pol $d$ 5) from Polistes dominulus venom led to the identification of single sensitization in $69 \%$ of 25 patients previously diagnosed as double positive to wasp venoms (Monsalve et al., 2012). Interestingly, Korošec et al. (2012) were able to identified 92\% (184/200) of patients previously diagnosed with Vespula venom allergy, using rVes $v 1$ and rVes $v 5$.

Phospholipase A1 is a major allergen widely distribute in social Hymenoptera venoms (Santos et al., 2011; Hou et al., 2016). Vespid phospholipases A1 (vPLA1s) are known to hydrolyze ester bonds of 1,2-diacyl-3-sn glycerophospholipids at the sn-1 position, converting these substrates into their corresponding lyso compounds and releasing fatty acids (Santos et al., 2007). During the envenoming process, vPLA1s are able to disrupt the phospholipid packing of biological membranes, causing severe hemolysis and leading to cardiac dysfunction and death in animals (Hou et al., 2016). The allergen showed direct hemolytic action against washed red blood cells (Santos et al., 2007). Molecular characterization and recombinant production of vPLA1s will improve strategies to reduce the toxic effects and immunologic reactions caused by this allergen during envenoming.
Similar to most wasps, proteomic and immunologic analyses of $P$. paulista venom led to identification of three major allergens: phospholipase A1 (Poly $p$ ), hyaluronidase (Poly $p 2$ ) and antigen 5 (Poly $p$ 5) (Santos et al., 2010). Purification, molecular and functional characterization of nPoly $p 1$ showed that this allergen is a non-glycosylated protein of $\sim 34 \mathrm{kDa}$, identified as a lipase of the GX class (Santos et al., 2007). Native Poly $p 1$ is $53-82 \%$ identical to the phospholipases of wasp species from the Northern Hemisphere. The protein structure has the $\alpha / \beta$ fold common to many lipases: a core consisting of a tightly packed $\beta$-sheet composed of a sixstranded parallel and one anti-parallel $\beta$-strand, surrounded by four $\alpha$-helices. A proteomic study from Santos et al. (2011) suggested that nPoly $p 1$ exists as a mix of multiple forms that show different levels of reactivity with sera of $P$. paulista venom-allergic patients.

To date, only the Poly $p 2$ has been cloned and expressed, using the prokaryotic system E. coli BL21 (DE3) (Justo-Jacomini et al., 2014). Recombinant Poly $p 2$ was produced as an insoluble protein, solubilized and later purified. High levels of IgE-reactivity were detected when sera from patients previously diagnosed with $P$. paulista venom allergy were tested against this protein.

Here, we describe the heterologous expression, purification and sIgE-mediated immunodetection of rPoly $p$ 1. IgE-reactivity of sera from sensitized patients to the recombinant allergen was compared with the recognition profile of its native counterpart ( $n$ Poly $p 1$ ) and crude venom extract. Our results show that rPoly $p 1$ represents a good candidate for improvement of $P$. paulista venom allergyspecific diagnosis.

\section{Materials and methods}

\subsection{Allergic patients' sera}

Sera from $10 P$. paulista venom-sensitized patients, regardless of sex or age, were obtained from the Ambulatório de Anafilaxia of the Hospital das Clínicas, Faculdade de Ciências Médicas, Universidade Estadual de Campinas-UNICAMP. The study was approved by the Ethics Committee of FCM-UNICAMP under $n^{\circ} 187 / 2006$. Informed consent was obtained in written form from all participants of the study, and participation was voluntary. Upon confirmation of positive intradermal reaction to $P$. paulista venom extract, the sera samples were collected and store at $-80^{\circ} \mathrm{C}$ until use. Five sera from non-sensitized patients were used as negative controls.

\subsection{Polybia paulista venom}

P. paulista nests were captured around or within the campus of Universidade Estadual Paulista "Júlio de Mesquita Filho" (UNESP), Rio Claro, SP, Brazil. The collected wasps were immediately anesthetized at low temperature $\left(-80^{\circ} \mathrm{C}\right)$, and their venom glands were extracted using sterile tweezers. For nPoly $p 1$ purification, the glands (4000) were washed with sterile water and suspended in a protease inhibitor mix (2 $\mathrm{mM}$ Leupeptine and $1 \mathrm{mM}$ phenylmethylsulfonyl fluoride; SIGMA, USA). The gland suspensions were then macerated, washed three times with the protease inhibitor mix and centrifuged at $10,000 \mathrm{~g}$ for $15 \mathrm{~min}$ at $4{ }^{\circ} \mathrm{C}$. The supernatant 
was collected, lyophilized and stored at $-80^{\circ} \mathrm{C}$ until use.

\subsection{RNA extraction and $C D N A$ synthesis}

For total RNA purification, 200 venom glands were washed in milli-Q sterile water, suspended in $200 \mu \mathrm{L}$ of Trizol ${ }^{\circledR}$ (Life Technologies, USA), macerated, and then stored for 7 days at $-80^{\circ} \mathrm{C}$. After RNA extraction, the first-strand cDNA synthesis was performed using $1 \mu \mathrm{g}$ of total RNA, the oligo dT-primer 5'- GGC CAC GCG TCG ACT AC(T) 17 -3' adapter (Gibco-Life Technologies, USA), and the ImProm-II Reverse Transcription kit (Promega,USA) according to manufacturer's instructions.

\subsection{Poly $p 1$ coding sequence amplification and cloning}

Primers for Poly $p 1$ coding sequence amplification were designed using data provided by Santos et al. (2007) (GenBank; access numbers: EF101736.1 and AM491805.1) and synthesized by Sigma-Aldrich (USA). Restriction sites (underlined) for NdeI and EcoRI were included in the forward (5'CTT A CATATG CTG ATT CCG GAA TGC CCG TTT AAC 3') and reverse (5'AGT G GAATTC TTA AAG TTT AAT GCC CTC GTT ATG ACA ATA AG 3') primers, respectively. PCR was performed as described by Justo-Jacomini et al. (2013), with minor modifications. Using an Eppendorf Mastercycler (Eppendorf, Germany), the following conditions were applied: initial denaturation at $95{ }^{\circ} \mathrm{C}$ for $5 \mathrm{~min}$; 40 cycles of denaturation at $95{ }^{\circ} \mathrm{C}$ for $1 \mathrm{~min}$; annealing at $57.5{ }^{\circ} \mathrm{C}$ for $1 \mathrm{~min}$ and extension at $72{ }^{\circ} \mathrm{C}$ for $3 \mathrm{~min}$; and a final extension at $72{ }^{\circ} \mathrm{C}$ for $15 \mathrm{~min}$. PCR products were checked by electrophoresis in agarose $2 \%$, purified using IlustraTM GFXTM PCR DNA and Gel Band Purification (GE Healthcare, USA), and further cloned into $\mathrm{pCR}^{\circledR} 8 / \mathrm{GW} / \mathrm{TOPO}^{\circledR}$ vector from the $\mathrm{pCR}^{\circledR} 8 / \mathrm{GW} / \mathrm{TOPO}^{\circledR}$ Cloning Kit (Invitrogen, USA) as per the manufacturer's instructions.

Immediately, One Shot ${ }^{\circledR}$ Mach1TMT1R chemically competent E. coli cells were reared in SOC Medium (2.0\% Tryptone, $0.5 \%$ yeast extract, $10 \mathrm{mM} \mathrm{NaCl}, 2.5 \mathrm{mM} \mathrm{KCl}, 10 \mathrm{mM} \mathrm{MgCl}_{2}, 10 \mathrm{mM} \mathrm{MgSO}_{4}$, and $20 \mathrm{mM}$ glucose) and transformed by thermal shock. Transformed cells were plated on Luria-Bertani agar (1.0\% Tryptone, $0.5 \%$ yeast extract and $1.0 \% \mathrm{NaCl}, \mathrm{pH} 7.0$ ) containing $100 \mu \mathrm{g} / \mathrm{mL}$ spectinomycin and incubated overnight at $37^{\circ} \mathrm{C}$. Vector preparations containing Poly $p 1$ coding sequence were obtained from a 5-mL culture of transformed clones, using the QIAprep ${ }^{\circledR}$ Spin miniprep kit (Qiagen, Germany) following the manufacturer's protocol, and were further analyzed by restriction digestion with Eco RI enzyme (Promega, USA).

\subsection{Gene sequencing}

Sequencing reactions were performed using the gene-specific primers described above (2.4) as well as forward (GW1: 5' GTT GCA ACA AAT TGA TGA GCA ATG C 3') and reverse (GW2: 5' GTT GCA ACA AAT TGA TGA GCA ATT A $3^{\prime}$ ) primers from the $\mathrm{pCR}^{\circledR} 8 / \mathrm{GW} /$ TOPO $^{\circledR}$ vector (Invitrogen, USA). Sequencing was conducted in an Applied Biosystems 3730 sequencer at the Center for Social Insects Studies (CEIS), Univ. Estadual Paulista "Júlio de Mesquita Filho" (UNESP), Rio Claro, SP, Brazil. Sequences were analyzed and edited using the DNASTAR ${ }^{\circledR}$ Lasergene Sequence Analysis software (http:// www.dnastar.com/t-allproducts.aspx).

\subsection{Heterologous expression and solubilization}

Plasmid constructs containing the Poly $p 1$ coding sequence were double-digested with Eco RI and Nde I restriction enzymes and sub-cloned into a pET-28a vector. The sequence was cloned in frame with the $\mathrm{N}$-terminal $6 \mathrm{xHis}$ tag of the commercial vector to facilitate rPoly $p 1$ purification through immobilized metal affinity chromatography. The pET-28a_poly $p 1$ plasmid construct was then used to transform E. coli XL1 Blue \{recA1 endA1 gyrA96 thi-1 hsdR17 supE44 relA1 lac [F proAB lacIqZDM15 Tn10 (Tetr)]\} (Stratagene, USA). The obtained vector preparations were used to transform the E. coli BL21 (DE3) chemically competent cells [FompThsdSB (r-B mB-) gal demD(srl-recA) 306:Tn10(DE3)] (Novagen, USA).

Heterologous expression was performed using the protocol described by Justo-Jacomini et al. (2014), with minor modifications. Briefly, $5 \mathrm{~mL}$ of LB medium supplemented with kanamycin (30 $\mu \mathrm{g} /$ $\mathrm{mL}$ ) was inoculated with transformed clones and grown overnight at $37{ }^{\circ} \mathrm{C}$ and $200 \mathrm{rpm}$ (Orbital Shaker Tecnal, Model TE-420). The pre-inoculums were then used to inoculate $50 \mathrm{~mL}$ of fresh medium. Once the culture reached a $\mathrm{DO}_{600}=0.4-0.6$, expression was induced by adding isopropyl $\beta$-D-1- thiogalactopyranoside (IPTG, Invitrogen, EUA) to a final concentration of $1 \mathrm{mM}$. Induced cultures were incubated at different temperatures $\left(37,30,25\right.$ and $\left.20^{\circ} \mathrm{C}\right)$. For each temperature, samples of $2.0 \mathrm{~mL}$ were collected at several times post-induction $(5,6,78,9,10$, and $24 \mathrm{~h})$. Cells were then collected, suspended in $200 \mu \mathrm{L}$ of lysis buffer $\left(50 \mathrm{mM} \mathrm{NaH}_{2} \mathrm{PO}_{4} \mathrm{pH}\right.$ 8.0, $300 \mathrm{mM} \mathrm{NaCl}, 20 \mathrm{mM}$ imidazole) and lysed by sonication in ice (six pulses of $1 \mathrm{~min}$, with $1 \mathrm{~min}$ between each pulse). Cell lysates were then centrifuged $\left(8000 \mathrm{~g}, 15 \mathrm{~min}, 4^{\circ} \mathrm{C}\right)$, and the pellet and supernatant were analyzed by SDS-PAGE to determine the best expression conditions and levels of soluble and insoluble expression.

For large-scale protein production, $5 \mathrm{~mL}$ of a pre-inoculum was added to $100 \mathrm{~mL}$ of LB medium. After induction with $1 \mathrm{mM}$ IPTG, bacterial culture was incubated at $20^{\circ} \mathrm{C}, 250 \mathrm{rpm}$ for $10 \mathrm{~h}$. Then, cells were collected by centrifugation ( $4000 \mathrm{~g}, 15 \mathrm{~min}$ and $4{ }^{\circ} \mathrm{C}$ ), suspended in $15 \mathrm{~mL}$ of lysis buffer and lysed; the insoluble fraction was collected by centrifugation $\left(8000 \mathrm{~g}, 15 \mathrm{~min}, 4^{\circ} \mathrm{C}\right)$. The pellet was further solubilized overnight at $4{ }^{\circ} \mathrm{C}$, with gentle agitation using $10 \mathrm{~mL}$ of a solubilization buffer $(50 \mathrm{mM}$ Tris- $\mathrm{HCl} 0.5 \mathrm{M} \mathrm{NaCl}$, $20 \mathrm{mM}$ imidazole and $8 \mathrm{M}$ urea, $\mathrm{pH}$ 8). Soluble fractions were then collected after centrifugation $\left(7500 \mathrm{~g}, 15 \mathrm{~min}, 4^{\circ} \mathrm{C}\right)$, analyzed by SDS-PAGE and stored at $4{ }^{\circ} \mathrm{C}$ until use.

\subsection{Recombinant allergen purification}

For rPoly $p 1$ purification, $10 \mathrm{~mL}$ of the soluble fraction were filtered with $0.22 \mu \mathrm{m}$ sterile filter and applied to a commercial prepacked column, HisTrap HPтм $\left(\mathrm{Ni}^{2+}\right.$ Sepharose ${ }^{\mathrm{TM}}$ High Performance; GE Healthcare, Sweden), coupled with a peristaltic pump (Pharmaceutical Biotech, Sweden). The column was then washed with $10 \mathrm{~mL}$ (10X-column volume) of each washing buffer (50 mM Tris- $\mathrm{HCl} \mathrm{pH} \mathrm{8,} \mathrm{containing} 0.5 \mathrm{M} \mathrm{NaCl}, 8 \mathrm{M}$ urea, and with increasing 50,75 and $100 \mathrm{mM}$ imidazole). Elution of His-tagged rPoly $p 1$ was performed with $10 \mathrm{~mL}$ of elution buffer $(50 \mathrm{mM}$ Tris- $\mathrm{HCl} 0.5 \mathrm{M}$ $\mathrm{NaCl}, 8 \mathrm{M}$ urea, $200 \mathrm{mM}$ imidazole; pH 8). Five fractions of $2 \mathrm{~mL}$ were collected at a flow rate of $1 \mathrm{ml} / \mathrm{min}$. All fractions collected during the purification process were further analyzed by (15\%) SDSPAGE.

\subsection{Purification of native phospholipase A1 from P.paulista venom}

The freeze-dried venom (23 mg of total protein) was solubilized in $50 \mathrm{mM}$ sodium acetate buffer $\mathrm{pH} 5.2$ and submitted to a cationexchange chromatography in a Hiprep FF CM column $(16 \times 10 \mathrm{~mm}$, $20 \mathrm{~mL}$; GE Healthcare) previously equilibrated with the same buffer and coupled with an Akta-FPLC system. Elution was accomplished by a linear gradient of $0-1 \mathrm{M} \mathrm{NaCl}$. Fractions of $5 \mathrm{~mL}$ were collected at a flow rate of $2 \mathrm{~mL} / \mathrm{min}$, and the elution was monitored by measuring the absorbance at $280 \mathrm{~nm}$. 


\subsection{SDS-polyacrylamide gel electrophoresis}

SDS-PAGE was performed according to Laemmli (1970), using a Mini-Protean ${ }^{\circledR}$ Tetra Cell System (BioRad). A prestained standard High-Range Rainbow Molecular Weight Marker (GE Healthcare, USA) and a PageRuler Prestained Protein Ladder (Thermo Scientific, USA) were loaded for SDS-PAGE and western blot analyses, respectively. After running, the gels were stained with either Coomassie Brilliant Blue R-250 (CBB) or silver stain (Fermentas, Germany).

\subsection{Protein quantification}

Protein fractions and purified native or recombinant allergens were quantified using the modified Bradford method and bovine serum albumin (Sigma, USA) as a standard (Sedmak and Grossberg, 1977).

\subsection{Enzymatic activity of phospholipase A1}

Phospholipase A1 enzymatic assays were performed using the EnzChek ${ }^{\circledR}$ phospholipase A1 assay kit (Invitrogen, USA) following the manufacturer's instructions. The measurement of all fractions collected after the cation-exchange chromatography was performed in triplicate.

\subsection{IgE-binding analyses}

Proteins submitted to SDS-PAGE gels were transferred to a $0.22 \mu \mathrm{m}$ nitrocellulose membrane using a semi-dry system (TransBlot $^{\circledR}$ SD Semi-Dry Electrophoretic Transfer Cell, Bio-Rad, USA), and the transfer efficiency was assessed by staining the gel with Coomassie Blue G-250 (Sigma, USA). Prior to the addition of patients' sera, membranes were blocked with $20 \mathrm{mM}$ Tris- $\mathrm{HCl} 150 \mathrm{mM} \mathrm{NaCl}$
pH 7.4, with 0.5\% Tween-20 (Sigma-Aldrich, USA) (TBS-T wash solution) and $3 \%$ non-fat dried milk (block solution). The membrane-blocking procedure was performed for $1 \mathrm{~h}$ of incubation at room temperature under slow agitation on a Rocker II ${ }^{\mathrm{TM}}$ platform mixer (Boekel Scientific, USA). After the wash (3 times with TBS-T), the membranes were transferred to a mini PROTEAN ${ }^{\circledR}$ II multiscreen apparatus (Bio-Rad, USA). Four hundred microliters of each patient's sera (diluted at 1:50 in a solution of TBS-T with $1 \%$ non-fat dried milk) were transferred to the individuals' channels. The system containing the membrane and the sera samples was incubated overnight at room temperature under slow agitation on a Rocker II ${ }^{\mathrm{TM}}$ platform mixer. Immunodetection was performed using antihuman IgE ( $\varepsilon$-chain specific) peroxidase conjugate antibody (Sigma-Aldrich, USA) diluted at 1:5000 (TBS-T and 1\% non-fat dried milk), and the bands were visualized in Image Quant 400 (GE Healthcare, Sweden) using the chemiluminescent substrate Luminata $^{\mathrm{TM}}$ Forte Western HRP substrate (Millipore, USA).

\section{Results}

\subsection{Recombinant Poly $p 1$ coding sequence}

A unique $958 \mathrm{pb}$ consensus cDNA sequence was obtained and annotated (GenBank GI: HQ023233.1) after the sequencing of 10 poly $p$ 1_pCR ${ }^{\circledR} 8 / \mathrm{GW} / \mathrm{TOPO}^{\circledR}$ positive clones. Analyses of the generated sequence, using the Blastn tool (http://blast.ncbi.nlm.nih.gov/ Blast.cgi), showed the highest similarity with the phospholipase A1 coding sequence from Polistes dominulus (84\%) and Polistes annularis (82\%). The putative primary sequence of rPoly $p$ 1(ADT89774.1) (Fig. 1) showed 302 amino acids with 13 Cys residues, 12 of them potentially involved in disulfide-bridge formation (Cys6-Cys89, Cys181- Cys176, Cys224-Cys219, Cys295- Cys242, Cys270-Cys241, Cys268-Cys263), as suggested by Santos et al. (2007). Analyses of the primary sequence rPoly $p 1$, using the Compute $\mathrm{pI} / \mathrm{MW}$ tool

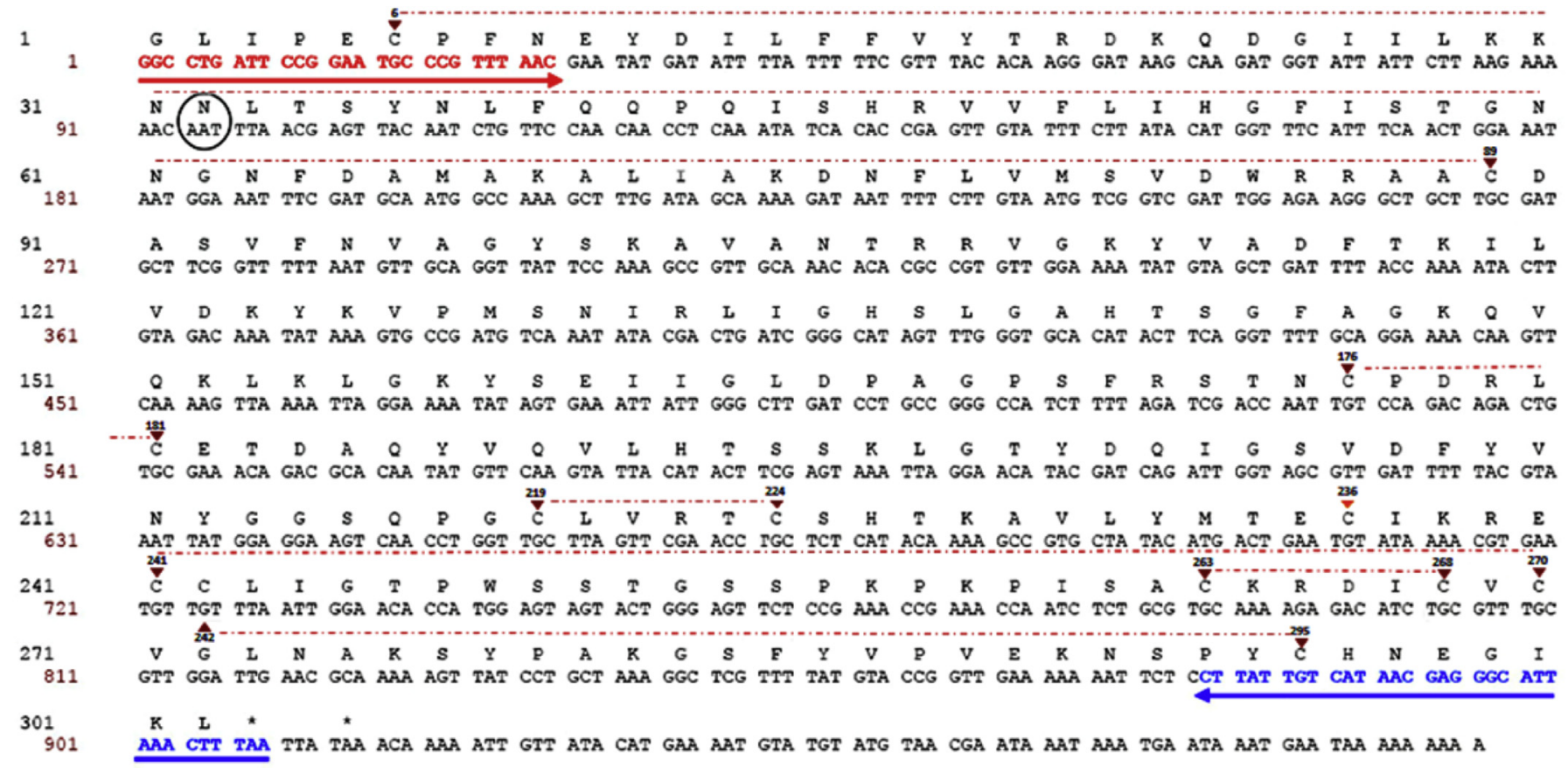

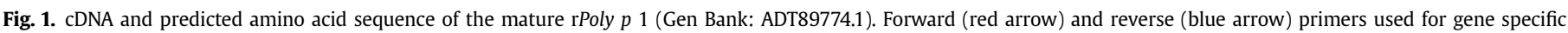

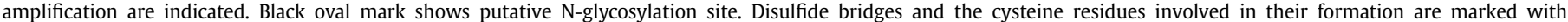

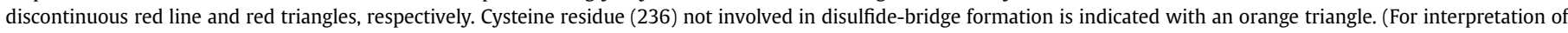
the references to colour in this figure legend, the reader is referred to the web version of this article.) 
(http://web.expasy.org/compute_pi/), showed a theorical pI value of 9.12 and a predicted molecular weight of $33.33 \mathrm{KDa}$.

Sequence alignment of rPoly $p 1$ (Fig. 2) showed low levels of identity with the venom phospholipases A1 of endemic wasps from the Northern Hemisphere-P. annularis (Pol a 1), GenBank: AAD52615.1 (71.9\%); V. vulgaris (Ves v 1), GenBank: AAB48072.1 (47.4\%); and V. germanica (Ves g 1), GenBank: CAJ28931.1 (53.8\%)and with the ant venom PLA1 from Solenopsis invicta (Sol $i$ 1), GI: NP_001291510.1 (27.2\%).

\subsection{Heterologous expression and solubilization of rPoly $p 1$ inclusion bodies}

Recombinant Poly $p 1$ was produced in E. coli BL21 (DE3) cells using pET-28a vector. Analysis of expression conditions showed that rPoly $p 1$ could be detected as soon as $5 \mathrm{~h}$ after induction with $1 \mathrm{mM}$ IPTG, growing at $20^{\circ} \mathrm{C}$ with agitation at $220 \mathrm{rpm}$ (Fig. 3). The highest level of the recombinant allergen $(706 \mathrm{mg} / \mathrm{L})$ was obtained $10 \mathrm{~h}$ post-induction using these growth conditions. In SDS-PAGE (8-20\%), rPoly $p 1$ appears as a band of $\sim 34 \mathrm{kDa}$, corresponding to the $34.1 \mathrm{kDa}$ theoretical molecular weight including the 6XHis tag, and similar to the $33.9 \mathrm{kDa}$ molecular weight of mature $\mathrm{nPoly} p$ 1 , as determined by Santos et al. (2007). No heterologous expression was detected at $37{ }^{\circ} \mathrm{C}, 30^{\circ} \mathrm{C}$ or $25^{\circ} \mathrm{C}$, and no increase in the expression levels was observed after $24 \mathrm{~h}$ post-induction (not shown).

SDS-PAGE analysis of soluble and insoluble fractions of $E$. coli BL21 (DE3) cells after lysis showed that rPoly $p 1$ is detected exclusively in the insoluble fraction (Fig. 4). No recombinant protein was detected in the supernatant of lysis.

\section{3. rPoly $p 1$ purification}

Recombinant Poly $p 1$ detected in the insoluble fraction after cell disruption was partially solubilized (30\%) with $50 \mathrm{mM}$ Tris- $\mathrm{HCl}$ $0.5 \mathrm{M} \mathrm{NaCl}, 20 \mathrm{mM}$ imidazole and $8 \mathrm{M}$ urea, $\mathrm{pH}$ 8. As rPoly $p 1$ contains a 6XHis tag, the HisTrap HPтм (GE Healthcare, Sweden) commercial prepacked column was used for recombinant allergen purification. Analyses of the collected fractions during purification showed that almost $100 \%$ of solubilized rPoly $p 1$ bonded to the $\mathrm{Ni}^{2+}$ Sepharose ${ }^{\mathrm{TM}}$ High Performance column (Fig. 5). The heterologous protein was eluted with $200 \mathrm{mM}$ imidazole. In SDS-PAGE analysis, a unique band with the expected MW $(\sim 34 \mathrm{kDa})$ was detected after elution. Further analysis of the SDS-PAGE results using the ImageJ (https://imagej.nih.gov/ij/) confirmed that rPoly $p 1$ was obtained with 95-99\% purity. Protein quantification showed that purified rPoly $p 1$ was obtained at a concentration of $212 \mathrm{mg} / \mathrm{L}$.

\section{4. nPoly p 1 purification and enzymatic activity}

The chromatographic profile of the crude venom extract (23 mg) obtained from Polybia paulista (Fig. 6) was similar to the profile described by Justo-Jacomini et al. (2013), showing 6 peaks designated $\mathrm{A}$ through $\mathrm{F}$. The major difference resided in the absence of the peak related to hyaluronidase activity. Low levels of Poly $p 2$ were further confirmed by SDS-PAGE and enzymatic activity analysis (data not shown).

The $\mathrm{nPoly} p 1$ was detected in peaks $\mathrm{E}$ (fractions 18-21) and $\mathrm{F}$ (fractions 26-29). Determination of PLA1 activity of pooled fractions from each peak, using the commercial system EnzChek ${ }^{\circledR}$ phospholipase A1 assay kit, showed $257 \mathrm{U} / \mathrm{mL}$ for peak E and 462

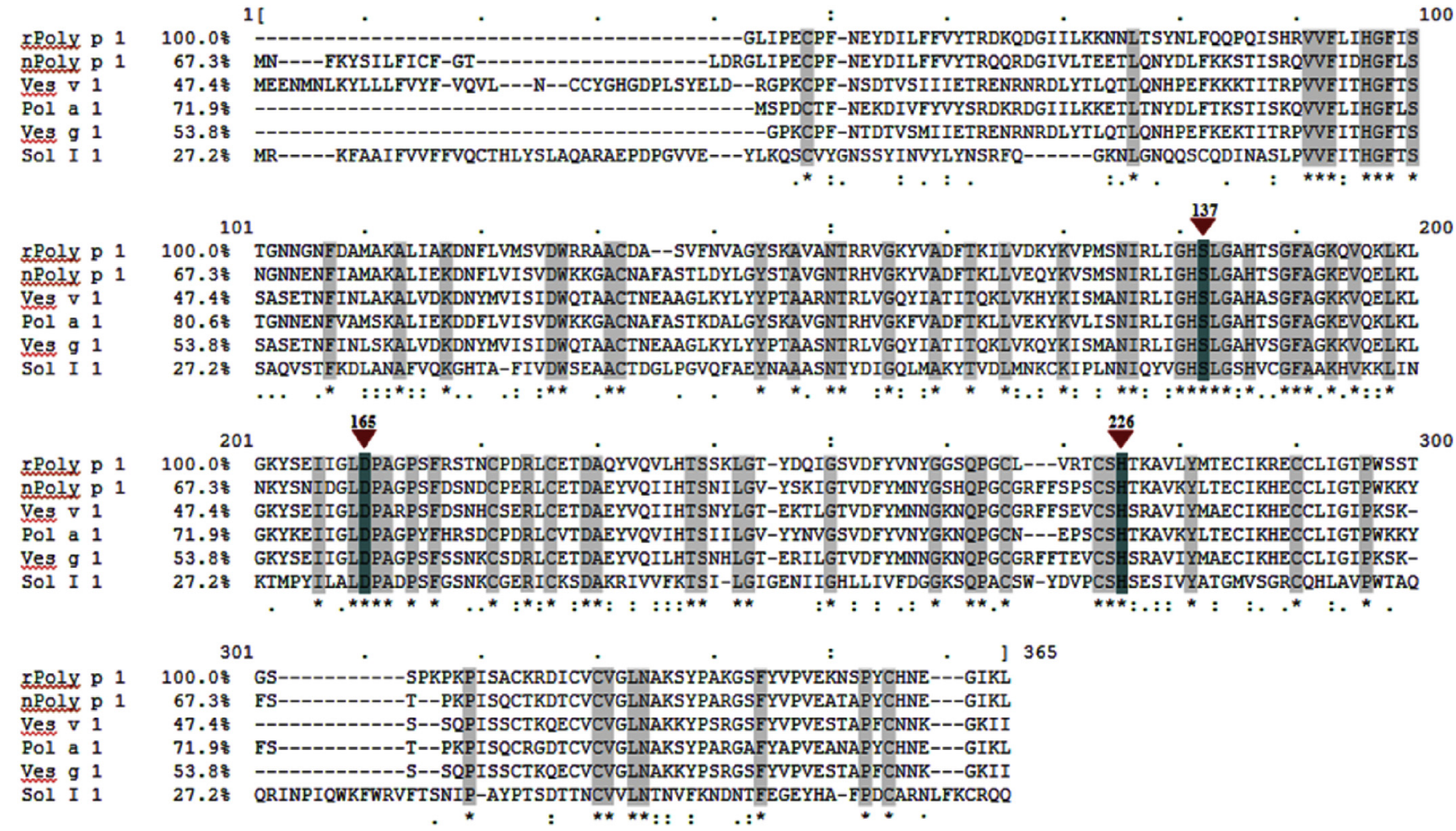

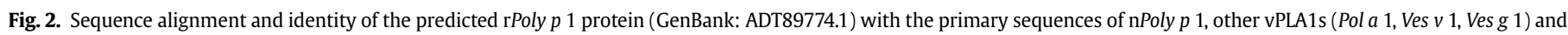

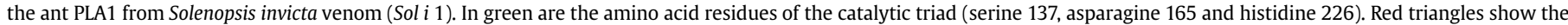

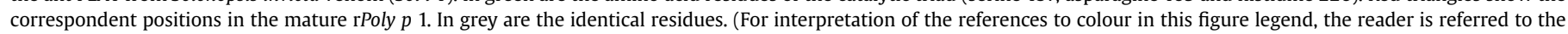
web version of this article.) 


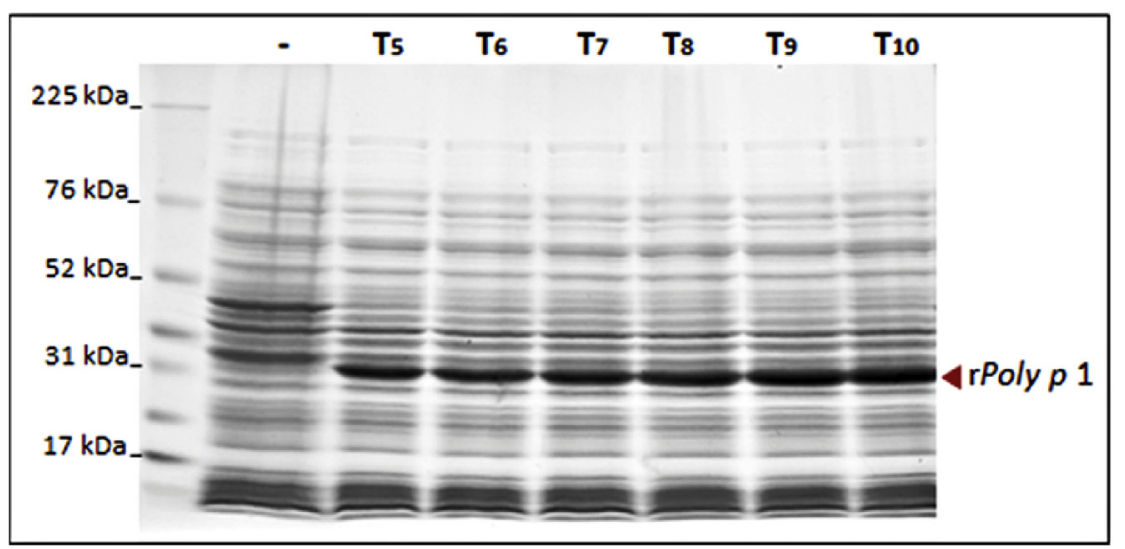

Fig. 3. SDS-PAGE (8-20\%) analysis showing the expression profile of rPoly $p 1$ in E. coli BL21 (DE3) cells at different times after induction $\left(20^{\circ} \mathrm{C}, 1 \mathrm{mM} \mathrm{IPTG).} \mathrm{T}_{5}-\mathrm{T}_{10}\right.$ represent hours after induction. ( - ) corresponds to a negative control (non-induced clone at $\mathrm{T}_{10}$ ).

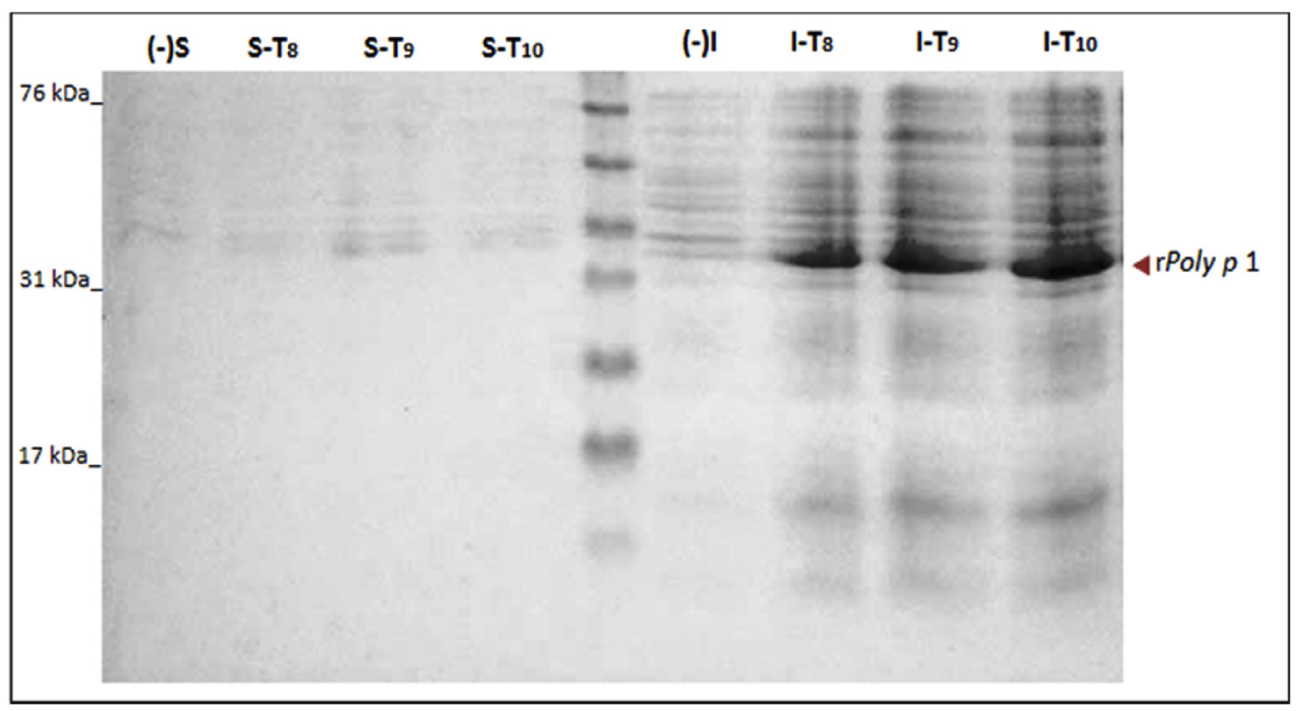

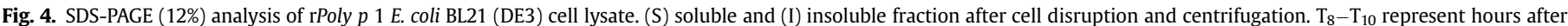
induction. (-) corresponds to a non-induced rPoly $\mathrm{p} 1$ clone at $\mathrm{T}_{10}$, used as a negative control.

$\mathrm{U} / \mathrm{mL}$ for peak $\mathrm{F}$, accounting for a phospholipase A1 activity recovery of $92 \%$. In SDS-PAGE analysis, purified nPoly $p 1$ from different fractions appeared as a unique band with the expected molecular weight and with a slight difference to rPoly $p 1$ due to the presence of the $6 \mathrm{xHis}$ tag in the recombinant form of the allergen (Fig. 7).

\subsection{Specific IgE-reactivity to recombinant and native Poly $p 1$}

Specific IgE-mediated immunodetection was analyzed against rPoly $p 1$, nPoly $p 1$ and crude venom extract, using sera from $10 P$. paulista venom-sensitized patients. Western blot assays showed similar reactions from the crude venom extract (Fig. 8A), nPoly $p 1$ (Fig. 8B) and rPoly $p 1$ (Fig. 8C) to the sera of patients previously diagnosed with $P$. paulista venom allergy using the ImmunoCAP system. No reaction occurred when E. coli BL21 (DE3) cell extracts were incubated with positive sera (not shown), nor when venom, native and recombinant allergen forms were incubated with sera from non-sensitized patients (negative controls) (Fig. 8A,B,C).

\section{Discussion}

Venoms from social Hymenoptera are complex mixtures of low molecular weight compounds (biogenic amines, lipids and carbohydrates, cationic peptides) and high molecular weight proteins, some with allergenic activity. Several venom allergens are glycosylated proteins and contain the so-called cross-reactive carbohydrate determinants (CCDs). Consequently, the use of crude extracts in allergy diagnosis is associated to cross-reactivity, miss-identification of the culprit venom, and finally, with non-specific sensitization of allergic patients submitted to SIT. Efforts to develop a molecular diagnosis of HVA based on the use of a panel of recombinant allergens obtained via different expression systems will improve allergy diagnosis and the SIT safety profile.

E. coli remains the most popular platform for recombinant protein expression and has been previously used for the production of several Hymenoptera venom allergens (Rosano and Ceccarelli, 2014; Spillner et al., 2014). In this work, we obtained high levels of rPoly $p 1$ expressed in E. coli BL21 (DE3) cells. The concentration of recombinant protein $(212 \mathrm{mg} / \mathrm{L})$, determined after purification with immobilized $\mathrm{Ni}^{2+}$ metal affinity chromatography, was similar to and even higher than those reported for other allergens 


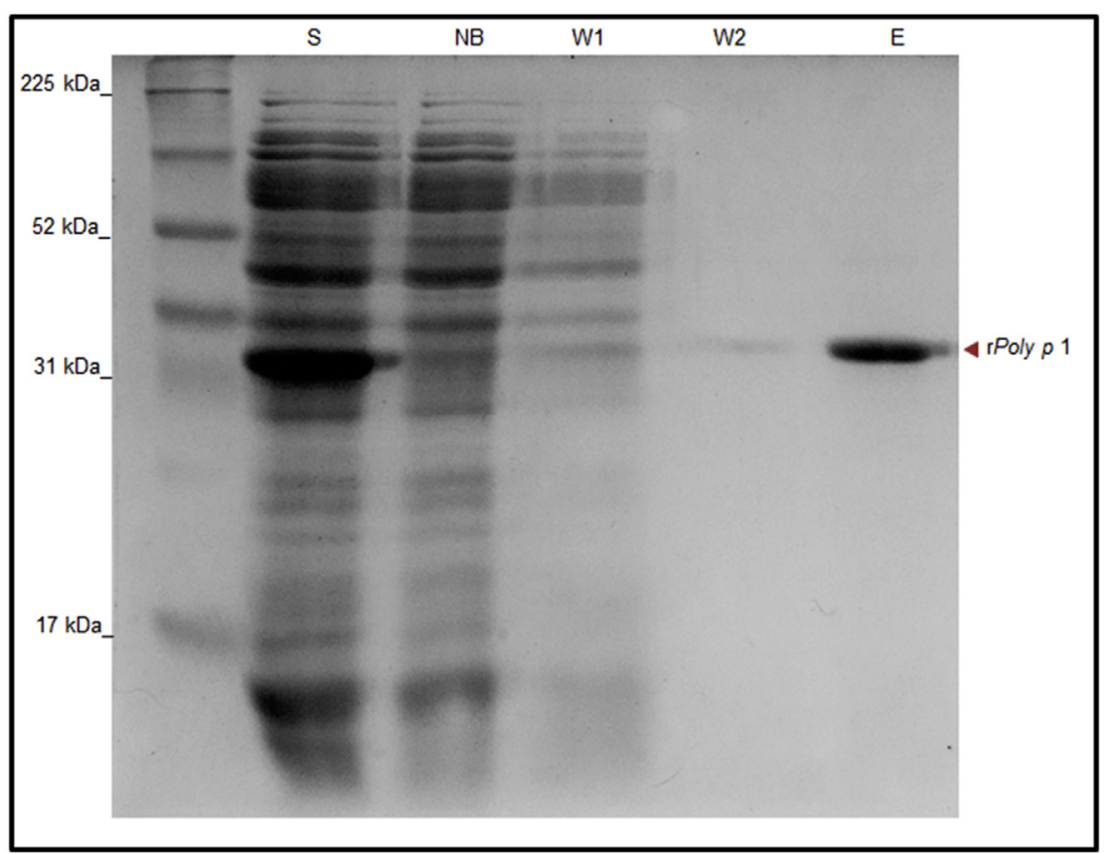

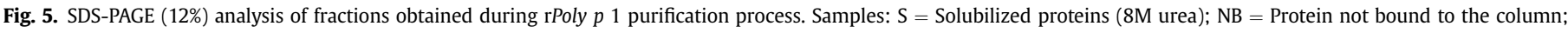
$\mathrm{W} 1-\mathrm{W} 2=$ fractions corresponding to column-washing steps with 75 and $100 \mathrm{mM}$ imidazole; $\mathrm{E}=$ fraction obtained after elution with $200 \mathrm{mM}$ imidazole.

expressed in E. coli (King et al., 1997; Skov et al., 2006; Lockwood et al., 2012) and other systems (Vinzón et al., 2010; Borodina et al., 2011). This agrees with the feasibility of the cell system and the purification procedure employed to express rPoly $p 1$ at high rates.

However, rPoly $p 1$ was produced exclusively in inclusion bodies. Heterologous protein expression in $E$. coli results in production of non-glycosylated molecules and usually prevents the formation of a disulfide bridge. Both factors may lead to protein misfolding, inactivation and aggregation. nPoly $p 1$ has been described as a nonglycosylated protein with six predicted disulfide bridges stabilizing its 3D structure (Santos et al., 2007). Analysis of the predicted rPoly p 1 primary sequence also showed the presence of 13 cysteine residues, 12 of which are potentially involved in the formation of six disulfide bridges (Fig. 1). The fast growth kinetics of $E$. coli, along with the referred high levels of rPoly $p 1$ expression and the possible failure to form the six predicted disulfide bridges, resulted in the production of an insoluble recombinant allergen. Further conditions during cultivation-lower temperature, inductor concentration and aeration rates-and co-expression with solubilityenhancement tags (Correa and Oppezzo, 2015) could be tested in order to obtain rPoly $p 1$ as a soluble protein using E. coli systems. Others authors have overcome the insolubility problem during recombinant protein expression by simply lowering the cultivation

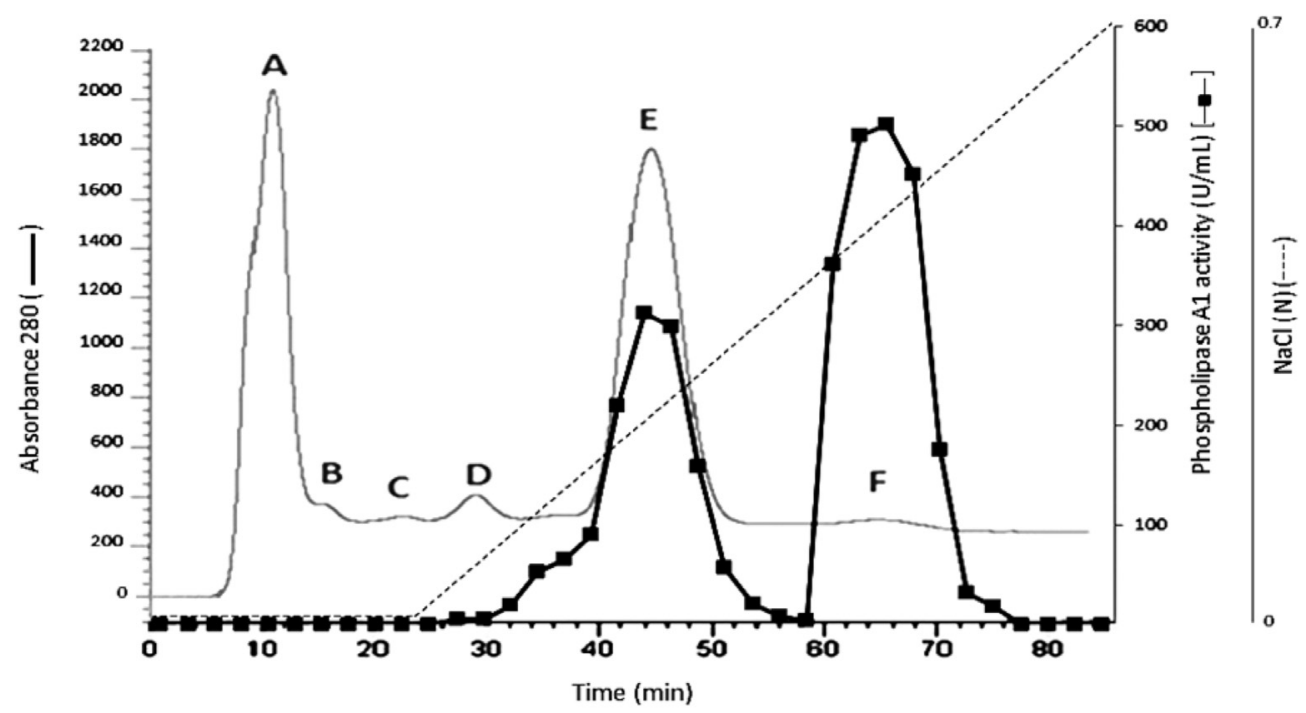

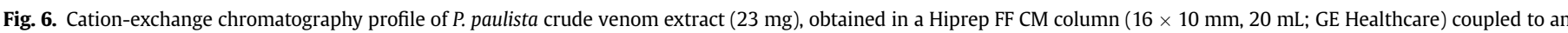

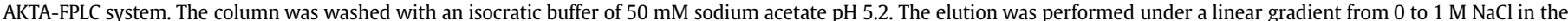

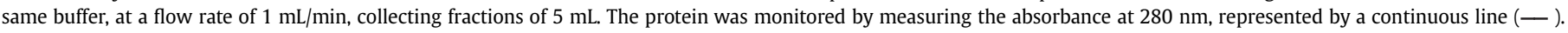
Phospholipase A1 enzymatic activity is represented with a shifting line-block $(\longrightarrow-$ ). 


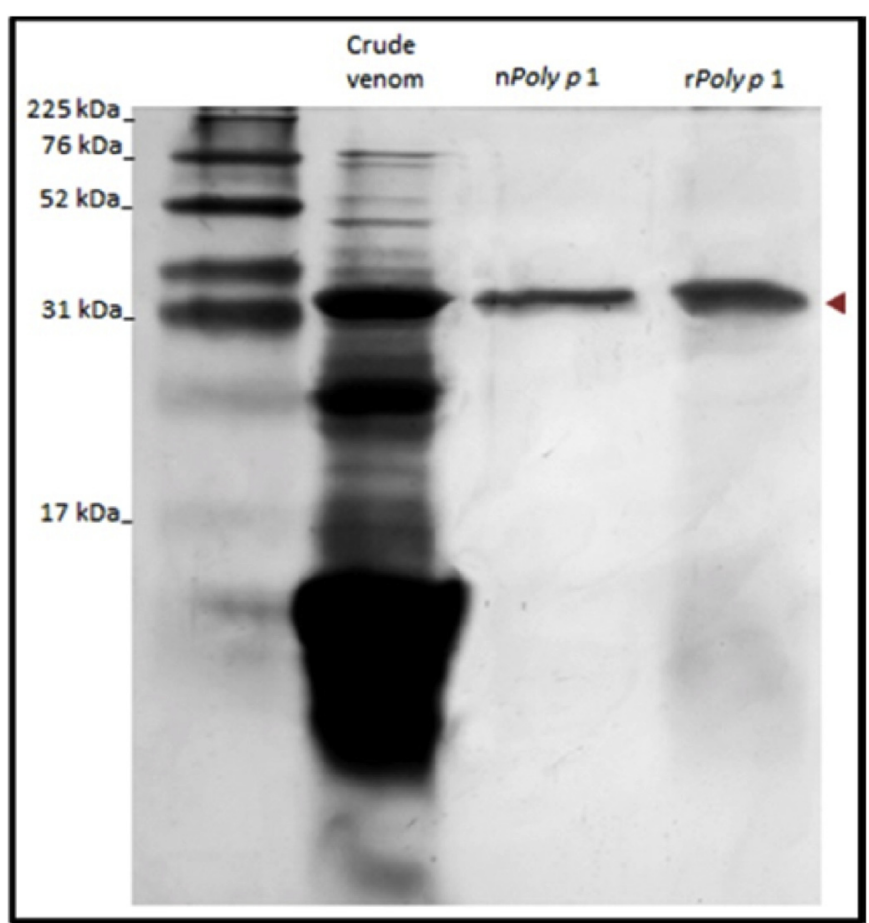

Fig. 7. SDS-PAGE (12\%) analysis of crude venom extract, nPoly $p 1$ and rPoly $p 1$. Gel was staining with silver-nitrate.

temperature $\left(6-10^{\circ} \mathrm{C}\right)$ (Song et al., 2012) or by the combination of different factors (San-Miguel et al., 2013; Vu et al., 2014; Sina et al., 2015). Finally, other expression platforms could be used to obtain rPoly $p 1$ as a soluble protein. For instance, the expression of Ves $v 5$ on yeast (Borodina et al., 2011) and insect cells (Seismann et al., 2010) circumvented insolubility problems of heterologous allergen production.

Previous attempts to obtain PLA1s from the venom of social Hymenoptera in E. coli also resulted in their expression as insoluble proteins. Obtained recombinant venom PLA1s showed lower binding to specific antibodies elicited by the native form of the allergens (King et al., 1997). These results were mainly associated with physicochemical as well as immunological differences between the renatured recombinant allergens and their native counterparts. In our case, similar levels of slgE-reactivity were detected in solubilized, but not refolded, rPoly $p 1$ and in denatured nPoly $p 1$ through western blot analysis (Fig. 8 B). These findings showed that linear IgE epitopes in the primary structure of nPoly $p$ 1 are involved in eliciting an allergic response in sensitized patients and are recognized in rPoly $p 1$. Similar behavior has been reported for either refolded or denatured recombinant allergens from dust mites (Tanyaratsrisakul et al., 2009; Floch et al., 2012) and social Hymenoptera venom (Skov et al., 2006; Justo-Jacomini et al., 2014), expressed on inclusion bodies in E. coli. Comparison of the recognition levels of $\mathrm{r}$ Poly $p 1$ with those of $\mathrm{nPoly} p 1$ and crude venom extract showed that solubilized rPoly $p 1$ could be used for the development of CRD of $P$. paulista venom allergy.

The one-step cation-exchange chromatographic procedure used in this work allowed complete purification of nPoly $p$ 1. This procedure diverged from the methodology described by Santos et al. (2007), in which the venom extract was initially submitted to a gel filtration chromatography, and then the fractions with phospholipase activity were pooled and submitted to cation-exchange chromatography. Interestingly, this work shows nPoly $p 1$ appearing in two peaks, and the fractions of each peak were analyzed with SDS-PAGE and with a highly specific fluorescence assay for phospholipase A1 enzymatic activity quantification. These results may be related to the heterogeneity of the venom material-obtained from individuals of different nests-used for chromatographic separation. Also, as was previously shown (Santos et al., 2011), $\mathrm{n}$ Poly $p 1$ exists as a mixture of multiple forms in $P$. paulista venom, which could be eluting at different $\mathrm{NaCl}$ concentrations during purification. Nevertheless, in SDS-PAGE analysis, all fractions with high levels of PLA1 activity showed a single band corresponding to nPoly $p 1$ molecular weight ( $34 \mathrm{kDa}$ ) (not shown). Further analysis of SDS-PAGE loaded with nPoly $p 1$ pooled fractions 18-21 from peak $\mathrm{E}$ and fractions 26-29 from peak $\mathrm{F}$, and staining with silver nitrate confirmed purity rates of 95-99\% (Fig. 7). Consider these results, a novel, efficient and economical purification process could be used for the purification of active nPoly $p 1$.

Recombinant PLA1s obtained from the venom of various social Hymenoptera species have been widely used in molecular allergy diagnosis for differentiation of true double sensitization from crossreactivity (Müller et al., 2012; Monsalve et al., 2012). The use of PLA1 has two remarkable advantages: as non-glycosylated proteins, they lack CCDs and thus reduce the incidence of crossreactivity. Furthermore, these major allergens are found in the venoms of wasps and ants, but not of honeybees; therefore, venom PLA1s represent useful markers to distinguish honeybee from wasp

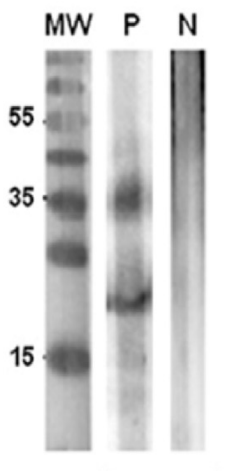

(A) Venom extract

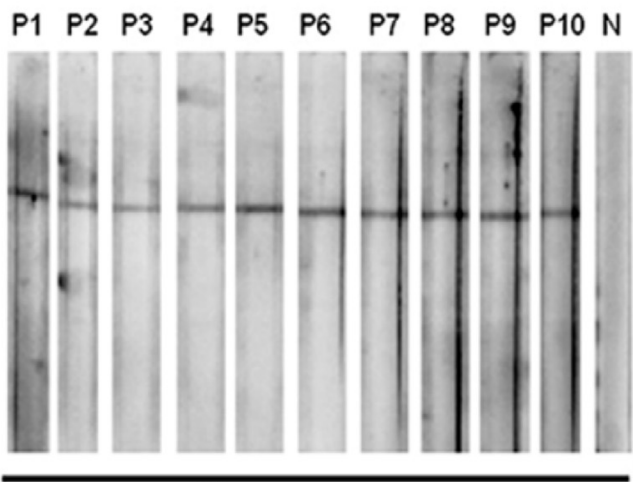

(B) nPoly p 1

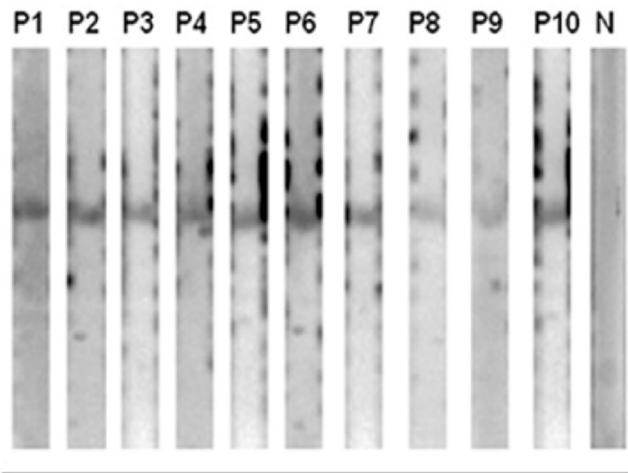

(C) rPoly p 1

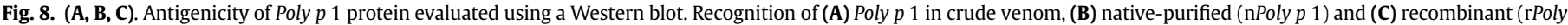

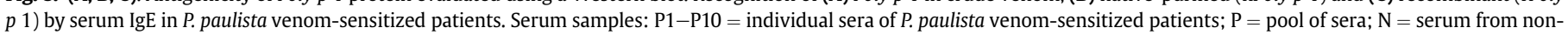
sensitized patient, as a negative control. The molecular weight marker $(\mathrm{kDa})$ is indicated. 
and/or ant venom allergy. In this context, and given the fact that only one (Poly $p 2$ ) of the three major allergens from $P$. paulista venom has thus far been cloned and evaluated as a candidate for the development of component-resolved diagnosis (Justo-Jacomini et al., 2014), we have cloned, expressed and analyzed the immunological activity of rPoly $p 1$ in the present study. As $100 \%$ of allergic patients' sera specifically recognized the rPoly $p 1$ to a similar extent as the purified nPoly $p 1$, the recombinant allergen appears to be a good candidate for the improvement of molecular diagnosis of $P$. paulista venom allergy. Recombinant Poly $\mathrm{p} 1$ could be used in combination with rPoly $p 2$ in order to detect patients that could be sensitized by either nPoly $p 1$ or nPoly $p 2$. The production of panels of major allergens from $P$. paulista venom-including Poly $p 5$, currently in expression process-will significantly reduce miss-identification of culprit venom during diagnosis and enhance the possibility of developing more effective, safe and less invasive strategies of SIT, aiding to improve the quality of life of $P$. paulista venom-allergic patients.

\section{Acknowledgments}

We acknowledge the financial support from FAPESP (São Paulo Research Foundation, Grant numbers: 2006/54799-6, 2014/139367) and from FUNDUNESP-UNESP (Fundação para o Desenvolvimento da Univ. Estadual Paulista, SP, Brazil; Grant number: 01197/ 10-DFP). The authors also thank to CAPES-DS (Coordenação de Aperfeiçoamento de Pessoal de Nível Superior), Postgraduate Program of Biological Sciences (Cellular and Molecular Biology) at UNESP, Rio Claro for providing Master and Doctoral scholarships to F.D.C.P and A.P.R. respectively, and to FAPESP (São Paulo Research Foundation, Proc.2009/51539-1) for Doctoral schoolarship to D.L.J.J.

\section{Transparency document}

Transparency document related to this article can be found online at http://dx.doi.org/10.1016/j.toxicon.2016.11.006

\section{References}

Bilò, M.B., 2011. Anaphylaxis caused by Hymenoptera stings: from epidemiology to treatment. Allergy 66, 35-37.

Bilò, M.B., Bonifazi, F., 2009. The natural history and epidemiology of insect venom allergy: clinical implications. Clin. Exp. Allergy 39, 1467-1476.

Borodina, I., Jensen, B.M., Wagner, T., Hachem, M.A., Søndergaard, I., Poulsen, L.K., 2011. Expression of enzymatically inactive wasp venom phospholipase A1 in Pichia pastoris. PLoS One 6, e21267.

Correa, A., Oppezzo, P., 2015. Overcoming the solubility problem in E. coli: available approaches for recombinant protein production. In: García-Fruitós, E. (Ed.), Insoluble Proteins: Methods and Protocols. Humana Press, Springer Inc., New York, pp. 27-45.

Floch, B.L., Bussières, L., Airouche, S., Lautrette, A., Bouley, J., Berjont, N., Horiot, S., Huet, A., Jain, K., Lemoine, P., Batard, T., Mascarell, L., Baron-Bodo, V., Tourdot, S., Nony, E., Moingeon, P., Chabre, H., 2012. Expression and characterization of natural-like recombinant Der p 2 for sublingual immunotherapy. Int. Arch. Allergy Immunol. 158 (2), 157-167.

Frick, M., Müller, S., Bantleon, F., Huss-Marp, J., Lidholm, J., Spillner, E., Jakob, T., 2015. rApi $\mathrm{m} 3$ and rApi $\mathrm{m} 10$ improve detection of honey bee sensitization in Hymenoptera venom-allergic patients with double sensitization to honey bee and yellow jacket venom. Allergy 70, 1665-1668.

Hou, M.H., Chuang, C.Y., Ko, T.P., Hu, N.J., Chou, C.C., Shih, Y.P., Ho, C.L., Wang, A.H.J., 2016. Crystal structure of vespid phospholipase A 1 reveals insights into the mechanism for cause of membrane dysfunction. Insect. biochem. Mol. Biol. 68, 79-88.

Jennings, A., Duggan, E., Perry, I.J., Hourihane, J.O., 2010. Epidemiology of allergic reactions to hymenoptera stings in Irish school children. Pediatr. Allergy Immunol. 21, 1166-1170.

Justo-Jacomini, D.L., Gomes-Moreira, S.M., Campos-Pereira, F.D., Zollner, R.L.,
Brochetto-Braga, M.R., 2014. Reactivity of IgE to the allergen hyaluronidase from Polybia paulista (hymenoptera, Vespidae) venom. Toxicon 82, 104-111.

Justo-Jacomini, D.L., Campos-Pereira, F.D., dos Santos-Pinto, J.R., dos Santos, L.D. Silva-Neto, A.J., Giratto, D.T., Palma, M.S., Zollner, R.L., Brochetto-Braga, M.R. 2013. Hyaluronidase from the venom of the social wasp Polybia paulista (Hymenoptera, Vespidae): cloning, structural modeling, purification, and immunological analysis. Toxicon 64, 70-80.

King, T.P., Lu, G., Gonzalez, M., Qian, N., Soldatova, L., 1997. Yellow jacket venom allergens, hyaluronidase and phospholipase: sequence similarity and antigenic cross-reactivity with their hornet and wasp homologs and possible implications for clinical allergy. J. Allergy Clin. Immunol. 98, 588-600.

Korošec, P., Valenta, R., Mittermann, I., Celesnik, N., Silar, M., Zidarn, M., Košnik, M., 2012. High sensitivity of CAP-FEIA rVes $v 5$ and rVes $v 1$ for diagnosis of Vespula venom allergy. J. Allergy Clin. Immunol. 129, 1406-1408.

Laemmli, U.K., 1970. Cleavage of structural proteins during the assembly of the head of bacteriophage T4. Nature 227, 680-685.

Lockwood, S.A., HaghiPour-Peasley, J., Hoffman, D.R., Deslippe, R.J., 2012. Identification, expression, and immuno-reactivity of Sol i 2 \& Sol $i 4$ venom proteins of queen red imported fire ants, Solenopsis invicta Buren (Hymenoptera: Formicidae). Toxicon 60 (5), 752-759.

Monsalve, R.I., Veja, A., Marques, L., Miranda, A., Fernandez, J., Soriano, V., Cruz, S. Domínguez-Noche, C., Sánchez-Morillas, L., Armisen-Gil, M., Guspi, R., Barber, D., 2012. Component-resolved diagnosis of vespid venom-allergic individuals: phospholipases and antigen 5 are necessary to identify Vespula or Polistes sensitization. Allergy 67, 528-536.

Müller, U.R., Schmid-Grendelmeier, P., Hausmann, O., Helbling, A., 2012. IgE to recombinant allergens Api $m$ 1, Ves v. 1, and Ves $v 5$ distinguish double sensitization from cross reaction in venom allergy. Allergy 67, 1069-1073.

Ollert, M., Blank, S., 2015. Anaphylaxis to insect venom allergens: role of molecular diagnostics. Curr. Allergy Asthma Rep. 15 (26), 1-11.

Perez-Riverol, A., Justo-Jacomini, D.L., Zollner, R.D.L., Brochetto-Braga, M.R., 2015 Facing hymenoptera venom allergy: from natural to recombinant allergens. Toxins 7, 2551-2570.

Rosano, G.L., Ceccarelli, E.A. 2014. Recombinant protein expression in Escherichia coli: advances and challenges. Front. Microbiol. 5 (172), 1-17.

San-Miguel, T., Pérez-Bermúdez, P., Gavidia, I., 2013. Production of soluble eukaryotic recombinant proteins in E. coli is favoured in early log-phase cultures induced at low temperature. SpringerPlus 2 (1), 1-4.

Santos, L.D., Menegasso, A.R.S., Santos-Pinto, J.R., Santos, K.S., Castro, F.M., Kalil, J.E., Palma, M.S., 2011. Proteomic characterization of the multiple forms of the PLAs from the venom of the social wasp Polybia paulista. Proteomics 11, 1403-1412.

Santos, L.D., Santos, K.S., de Souza, B.M., Arcuri, H.A., Cunha-Neto, E., Castro, F.M., Kalil, J.E., Palma, M.S., 2007. Purification, sequencing and structural characterization of the phospholipase A1 from the venom of the social wasp Polybia paulista (Hymenoptera, Vespidae). Toxicon 50, 923-937.

Santos, L.D., Santos, K.S., Pinto, J.R., Dias, N.B., de Souza, B.M., dos Santos, M.F. Perales, J., Domont, G.B., Castro, F.M., Kalil, J.E., Palma, M.S., 2010. Profiling the proteome of the venom from the social wasp Polybia paulista: a clue to understand the envenoming mechanism. J. Proteome Res. 9, 3867-3877.

Sedmak, J.J., Grossberg, S.E., 1977. A rapid, sensitive and versatile assay for protein using Coomassie brilliant blue G250. Anal. Biochem. 79, 544-552.

Seismann, H., Blank, S., Cifuentes, L., Braren, I., Grunwald, T., Ollert, M., Spillner, E. 2010. Recombinant phospholipase A1 (Ves $v$ 1) from yellow jacket venom for improved diagnosis of hymenoptera venom hypersensitivity. Clin. Mol. Allergy $8,1-8$.

Sina, M., Farajzadeh, D., Dastmalchi, S., 2015. Effects of environmental factors on soluble expression of a humanized anti-TNF- $\alpha$ scFv antibody in Escherichia coli. Adv. Pharm. Bull. 5 (4), 455-461.

Skov, L.K., Seppala, U., Coen, J.J.F., Crickmore, N., King, T.P., Monsalve, R., Kastrup, J.S. Spangfort, M.D., Gajhede, M., 2006. Structure of recombinant Ves v 2 at 2.0 angstrom resolution: structural analysis of an allergenic hyaluronidase from wasp venom. Acta Crystallogr. D. Biol. Crystallogr. 62, 595-604.

Song, J.M., An, Y.J., Kang, M.H., Lee, Y.H., Cha, S.S., 2012. Cultivation at $6-10^{\circ} \mathrm{C}$ is an effective strategy to overcome the insolubility of recombinant proteins in Escherichia coli. Protein Expr. Purif. 82, 297-301.

Spillner, E., Blank, S., Jakob, T., 2014. Hymenoptera allergens: from venom to venome. Front. Immunol. 5 (77), 1-7.

Tanyaratsrisakul, S., Malainual, N., Jirapongsananuruk, O., Smith, W.A., Thomas, W.R., Piboonpocanun, S., 2009. Structural and IgE binding analyses of recombinant Der $p 2$ expressed from the hosts Escherichia coli and Pichia pastoris. Int. Arch. Allergy Immunol. 151 (3), 190-198.

Vinzón, S.E., Pirpignani, M.L., Nowicki, C., Biscoglio de Jimenez-Bonino, M., 2010 Molecular cloning and expression in Pichia pastoris of a hypoallergenic antigen 5. Protein Expr. Purif. 73, 23-30.

Vu, T.T.T., Jeong, B., Yu, J., Koo, B.K., Jo, S.H., Robinson, R.C., Choe, H., 2014. Soluble prokaryotic expression and purification of crotamine using an N-terminal maltose-binding protein tag. Toxicon 92, 157-165. 